\title{
Leichtbau braucht Unterstützung
}

Der im Automobilbau immer wieder zitierte Wunsch nach dem „richtigen Werkstoff am richtigen Ort" ist ebenso berechtigt wie schwer zu erfüllen. Zu unterschiedlich sind die Materialparameter, die im einen Anwendungsfall geeignet sind, im anderen nur eine Ablehnung zulassen. Um eine sinnvolle Entscheidung zugunsten diverser metallischer oder kunststoffbasierter Lösungen zu ermöglichen, spielen nicht nur Materialeigenschaften eine Rolle, mit entscheidend sind vor allem Fragen zu Werkstoffverfügbarkeit, Kosten, Verhalten in der Produktion und Rezyklierbarkeit. Letztere gewinnt in Zeiten knapperer Ressourcen stetig an Bedeutung.

Im Volumensegment behauptet Stahl nach wie vor seine dominante Stellung. Doch findet das Leichtmetall Aluminium in Konstruktion und Entwicklung immer mehr Freunde. Als Schwerpunkt dieser Ausgabe von lightweight design beleuchten wir die Entwicklungen von Stahl, Aluminium und auch Magnesium als Leichtbauwerkstoff in Konkurrenz zu verstärkten Kunststoffen.

So befassen sich die Autoren des Fraunhofer IWU in Stuttgart mit dem Einsatz von hochfesten Stählen in reduzierten Wanddicken und deren Nachteilen für die Produktionstechnik sowie Lösungsansätzen für den Einsatz von Magnesium und hochfesten Aluminiumlegierungen.

Der Beitrag von Constellium beschreibt unter anderem, wie sich zwar ultrahochfeste, aber auch schwer formbare 7000er-Aluminiumlegierungen anschicken, auch im Automobil- sektor Fuß zu fassen und im Vergleich „den besten Kompromiss zwischen Gewichtseinsparungspotenzial und Kosten bieten."

Bei allen herausragenden Erfolgen, die der Leichtbau im Auto bislang ermöglichte - er allein kann es nicht richten. Ein moderner Materialmix allein wird nicht dazu führen, die bis 2020 vorgegebenen Emissionswerte von $95 \mathrm{~g} \mathrm{CO}_{2} / \mathrm{km} \mathrm{zu}$ erreichen. Auch nicht mit den bisherigen Antriebskonzepten, selbst wenn diese immer weiter optimiert werden. Im Gegenteil: Pkw werden immer größer und trotz verwendeter Leichtbaumaterialien tendenziell schwerer. Die Automobilindustrie klagt vorsorglich schon jetzt, dass die Grenzwerte zu streng sind, und setzt weiterhin auf Performance und Fahrspaß.

Das autonome Fahren wird unter anderem wegen ungeklärter IT-Sicherheit und rechtlicher Fragen eine Weile Zukunftsmusik bleiben; die Elektromobilität kommt wegen zu geringer Batteriekapazitäten und einer schlechten Ladeinfrastruktur nicht so recht voran. Gefragt sind völlig neu gedachte Verkehrskonzepte, bei denen nicht nur dem Leichtbau alle Erwartungen in Bezug auf die $\mathrm{CO}_{2}$-Verringerung auferlegt werden. Flankierend ließe sich nach heutiger Kenntnislage zum Beispiel auch wieder eine alte Forderung unserer „Umweltpartei“ wiederbeleben, ob ein nervenschonenderes Tempolimit nicht doch zu einer Verringerung der Emissionen (und Adrenalinwerte) führt - auch wenn bei den Grünen selbst davon keine Rede mehr ist.

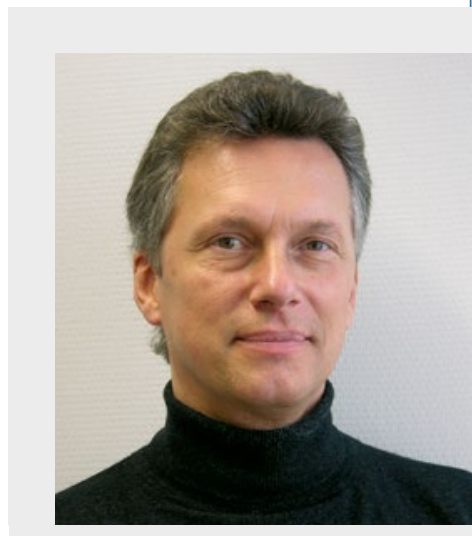

Dipl.-Ing. Ulrich Knorra Redakteur, ulrich.knorra@springer.com 